\title{
Combining tunable proteolytic sequences and a VEGF-mimetic peptide for the spatiotemporal control of angiogenesis within Elastin-Like Recombinamer scaffolds
}

Fernando González-Pérez ${ }^{1}$, Arturo Ibáñez-Fonseca ${ }^{1}$, Matilde Alonso ${ }^{1}$, José Carlos RodríguezCabello $^{1}$

${ }^{I}$ G.I.R. BIOFORGE (Group for Advanced Materials and Nanobiotechnology), CIBER-BBN, Edificio LUCIA, Universidad de Valladolid, Paseo Belén 19, 47011 Valladolid, Spain

Keywords: Elastin-like recombinamer, angiogenesis, VEGF, hydrogels, tunable proteolytic sequences

Corresponding author: Prof. José Carlos Rodríguez-Cabello, Edificio LUCIA, Paseo de Belén, 19, 47011, Valladolid, Spain. Email: roca@bioforge.uva.es

\section{$\underline{\text { Abstract }}$}

One of the main challenges in regenerative medicine is the spatiotemporal control of angiogenesis, which is key for the successful repair of many tissues, and determines the proper integration of the implant through the generation of a functional vascular network. To this end, we have designed a three-dimensional (3D) model consisting of a coaxial binary elastin-like recombinamer (ELR) tubular construct. It displays fast and slow proteolytic hydrogels on its inner and outer part, respectively, both sensitive to the urokinase plasminogen activator protease. The ELRs used to build the scaffold included crosslinkable domains to stabilize the structure and a conjugated VEGF-derived peptide (QK) to induce angiogenesis. The mechanical and morphological evaluation of the ELR hydrogels proved their suitability for soft tissue regeneration. In addition, in vitro studies evidenced the effect of the QK peptide on endothelial cell spreading and anastomosis. Moreover, immunohistochemical analyses after subcutaneous implantation of the ELR hydrogels in mice showed the induction of a low macrophage response that resolved over time. The implantation of the 3D model constructs evidenced the ability of the fast proteolytic sequence and the QK peptide to guide cell infiltration and capillary formation in the pre-designed arrangement of the constructs. These results set the basis for the application of this type of scaffolds in regenerative medicine, where spatiotemporally controlled vascularization will help in the promotion of an optimal tissue repair. 


\section{Introduction}

Successful tissue engineering and regenerative medicine (TERM) approaches aiming for the replacement of damaged tissues depend on the formation of a functional capillary network through angiogenesis. The development of these structures is a complex process that starts with the release of degrading proteases, followed by the activation of endothelial cells and vascular sprouting into the interstitial space [1-4]. One of the main issues in TERM is the spatiotemporal control of angiogenesis, necessary for the correct adaptation to the natural vascular architecture and for the creation of complex engineered vascular structures $[5,6]$. A potential solution is the development of protease-sensitive hydrogels that encapsulate and release bioactive molecules, such as vascular endothelial growth factor (VEGF) [1, 7]. However, this may lead to the burst release of the factors and to vascular malformations, such as immature networks or tumorigenesis [8-11]. Thus, novel strategies that enable the spatiotemporal control of cleavage kinetics and angiogenesis are needed.

To this end, protein-based biomaterials obtained through recombinant DNA technology arise as excellent candidates, due to their tunability and the precise control over their composition [12]. Specifically, elastin-like recombinamers (ELRs) are recombinant protein polymers based on the repetition of the Val-Pro-Gly-Xaa-Gly (VPGXG) pentapeptide, found in natural elastin, where $X$ can be any amino acid, except proline [13]. Moreover, ELRs exhibit a characteristic behaviour of phase separation in aqueous environments above the so-called transition temperature $\left(T_{\mathrm{t}}\right)$, which allows a cost-effective purification through cooling and heating cycles, a process known as inverse transition cycling (ITC) $[14,15]$. Interestingly, they can be chemically modified for their efficient crosslinking through fast, high-yield and orthogonal "click chemistry" reactions [16], as previously described [17]. Among them, the catalyst (copper)-free strain-promoted [3+2] azidealkyne cycloaddition (SPAAC), developed by Bertozzi and co-workers in 2004, has been commonly used with living cells due to its improved cytocompatibility $[18,19]$. This technique has reported the formation of biocompatible ELR hydrogels [20] that promoted the regeneration of different tissues, thanks to their bioinspired ECM-like nature and to the inclusion of bioactive sequences [21]. For instance, they have shown to enable the regeneration of bone [22], cartilage [23, 24], skeletal muscle [25], cornea [26] and cardiovascular tissues [27-29]. In addition, ELRs have demonstrated a non-immunogenic response, promoting the shift from type-1 macrophages ( $M 1$, pro-inflammatory) towards type-2 macrophages ( $M 2$, pro-regenerative), thus preventing fibrosis and inducing an improved healing of the damaged tissue [25]. Furthermore, recent works have described the development of ELR hydrogels that display proangiogenic factors to promote the formation of vessels [30], and others that contain protease- 
sensitive moieties in order to guide the process [31]. In the first case, an ELR hydrogel including Arg-Gly-Asp (RGD) motifs that promote cell adhesion and a covalently tethered VEGF-mimetic peptide, known as QK [32], induced the generation of functional capillaries in vivo [30]. This chemical conjugation approach has proved to be more efficient than non-crosslinked bearing peptides where a constant local concentration of the angiogenic agent does not exist, leading to non-reproducible results and undesired negative side effects [8-11]. On the other hand, protease-sensitive ELRs have been used to form scaffolds with specific configurations to control the cell invasion rate in a predesigned way [31]. For this purpose, two urokinase plasminogen activator (UPA)-sensitive sequences were encoded in different ELR molecules. These sequences differ in their cleavage kinetics, being slow (Asp-Arg-lle-Arg or DRIR)- and fast (Gly-Thr-Ala-Arg or GTAR)-proteolytic motifs. The use of ELR hydrogels including these domains allowed the formation of a 'sandwich' scaffold that guided cell invasion and migration, which occurred first in the fast-proteolytic layer and spread to the slow-proteolytic one from there [31]

Herein, we hypothesize that the combination of the aforementioned properties by including the pro-angiogenic QK peptide and the UPA-sensitive fast- and slow-proteolytic sequences in the same scaffold may synergistically contribute to the spatiotemporal control of angiogenesis in vivo. To assess this hypothesis, we developed a 3D model consisting of a coaxial binary ELR tubular scaffold where the tubes were designed to promote an oriented cell invasion and angiogenesis. Specifically, the external tube was built through the combination of the DRIR-ELR and the RGD-ELR (with slow-proteolytic and cell-adhesive RGD sequences, respectively). On the other hand, the internal tube was made of a mixture of the fast-proteolytic GTAR-ELR and the RGD-ELR. In addition, the QK peptide was covalently tethered to the RGD-ELR, thus allowing us to evaluate its pro-angiogenic effect when included in both parts or in the internal one only. We propose that this 3D model will allow to invert the natural pattern of the angiogenesis process, which appears first in the external layer, to depict a unidirectional anisotropic pattern in which infiltration and vascularization takes place first in the internal tube following the pre-designed arrangement. To shed light into the behaviour of the ELR scaffolds, we performed morphological and mechanical studies to evaluate their physical properties as implantable hydrogels. Subsequently, we studied the effect of the ELR-QK hydrogels on human umbilical vein endothelial cells (HUVECs), in comparison with soluble VEGF. Finally, the 3D models (i.e. coaxial binary ELR tubular scaffolds), as well as their constituting tubes (i.e. the single ELR hydrogels), were implanted subcutaneously in mice. Histological and immunohistochemical analyses allowed us to assess the macrophage response and the ability of the scaffolds to promote a 
spatiotemporal control on the formation of functional microvasculature in vivo after 1, 3 and 6 weeks.

\section{Materials and methods}

\subsection{ELR synthesis, modification and characterization}

All the ELRs used in this work, namely GTAR-ELR, DRIR-ELR, RGD-ELR and VKV-ELR, have been previously described [19, 31], and their sequences can be found in Table S1 (Supporting Information). The GTAR-ELR, DRIR-ELR and RGD-ELR are based on the repetition of the $\left((V P G I G)_{2} \text { VPGKG(VPGIG) }\right)_{2}$ ) elastin-like block. Specifically, the GTAR-ELR and DRIR-ELR include fast (GTAR) and slow (DRIR) uPA-sensitive proteolytic sequences, respectively, whereas the RGDELR contains RGD domains that promote cell adhesion. On the other hand, the VKV-ELR is based on the repetition of the (VPGKG(VPGVG) $)_{5}$ ) block and lacks bioactive sequences within its backbone. Their bioproduction was performed as described elsewhere [33]. Briefly, the ELRs were expressed in Escherichia coli bacteria in a 15-L bioreactor and purified through ITC [15]. The aqueous solutions of the pure recombinamers were dialyzed against deionized water, filtered through $0.22 \mu \mathrm{m}$ membranes for sterilization and freeze-dried for stable storage.

As regards chemical modification, azide groups were tethered to the RGD-ELR, whereas cyclooctyne groups were conjugated to the GTAR-ELR, DRID-ELR and VKV-ELR to give the 'click'modified versions necessary for the orthogonal crosslinking and hydrogel formation $[18,19]$. On the other hand, a QK peptide bearing a dibenzocyclooctyne (DBCO) group in the amino terminal group (H-Cys-[(3-succinimido-propionyl)-B-Ala-DBCO]-Lys-Leu-Thr-Trp-Gln-Glu-Leu-Tyr-GlnLeu-Lys-Tyr-Lys-Gly-Ile-OH trifluoroacetate salt; Bachem) was tethered via 'click' reaction to the azide groups of the modified RGD-ELR, thus giving the RGD-QK-ELR. The stoichiometry of this reaction was 0.5:1 (QK:ELR), so half of the RGD-QK-ELR molecules comprised the bioconjugated peptide. Furthermore, a fluorescent version was fabricated by conjugating the Cyanine 5 (Cy5) molecule (1:1, Cy5:ELR), a far-red fluorophore, via "nucleophilic substitution" to the RGD-QKELR. In brief, a solution of NHS-Cyanine5 (Jena Bioscience) was added to a solution of RGD-N3QK (1:1 Cy5:ELR), both in ultrapure water, and the resulting mixture was stirred for $18 \mathrm{~h}$ at $4^{\circ} \mathrm{C}$. Then, the solution was dialyzed against ultrapure water, the $\mathrm{pH}$ adjusted to 7 , filtered through $0.22 \mu \mathrm{m}$ membranes for sterilization and freeze-dried to yield a far-red fluorescent recombinamer (RGD-N3-QK-Cy5). This same purification protocol was applied for all the modified recombinamers. The characterization was performed by Fourier transform infrared spectroscopy (FTIR), proton nuclear magnetic resonance $\left({ }^{1} \mathrm{H}-\mathrm{NMR}\right)$, differential scanning 
calorimetry (DSC) and matrix-assisted laser desorption/ionization time-of-flight mass spectrometry (MALDI-TOF) (Fig. S1-S8, Supporting Information).

\subsection{Hydrogel formation}

Cyclooctyne- (GTAR-ELR, DRIR-ELR or VKV-ELR) and azide-modified (RGD-ELR or RGD-QK-ELR) recombinamers were dissolved (each ELR in a separate tube) in phosphate buffer saline (PBS 1X, $\mathrm{pH} 7.2$ ) at $50 \mathrm{mg} / \mathrm{mL}$ through an overnight $(\mathrm{O} / \mathrm{N})$ incubation at $4{ }^{\circ} \mathrm{C}$. Subsequently, different combinations of cyclooctyne- and azide-modified ELRs were mixed at a 1:1 volume ratio, giving the corresponding Fast (GTAR-ELR + RGD-ELR), Fast-QK (GTAR-ELR + RGD-QK-ELR), Slow (DRIR$E L R+R G D-E L R)$, Slow-QK (DRIR-ELR + RGD-QK-ELR), Non-degradable (VKV-ELR + RGD-ELR) and Non-degradable-QK (VKV-ELR + RGD-QK-ELR) ELR hydrogels (Table S2, Supporting Information). The mixtures were incubated at $4{ }^{\circ} \mathrm{C}$ (below the $T_{\mathrm{t}}$ of every ELR used) for a minimum of $30 \mathrm{~min}$ to obtain a homogeneous hydrogel, unless otherwise stated.

\subsection{Mechanical and morphological properties of the ELR hydrogels}

For the mechanical measurements, hydrogels with a volume of $260 \mu \mathrm{L}$ were prepared in a $12 \mathrm{~mm}$ diameter mold. Oscillatory measurements were done using parallel plates geometry in an AR-2000ex rheometer (TA instruments) at physiological temperature $\left(37^{\circ} \mathrm{C}\right)$, and with a gap of approximately $1000 \mu \mathrm{m}(\mathrm{n}=3)$. The complex elastic modulus of the ELR hydrogels was obtained through a time-sweep experiment ( $1 \%$ strain, $1 \mathrm{~Hz}$ frequency), keeping the samples in PBS $1 \mathrm{X}$ to avoid dehydration and shrinking during the study. All the measurements were performed in the linear viscoelastic region, as shown in Fig. S9-S14 (Supporting Information).

The pore size and morphology of the ELR hydrogels were determined by scanning electron microscopy (SEM). For this, the formed hydrogels were frozen by immersion in liquid nitrogen, cryo-fractured and lyophilized. Samples were coated with sputtered gold and SEM images were obtained in a FEI Quanta 200FEG (Thermo Scientific). The morphology was analyzed using the ImageJ software [34].

\subsection{Hydration analysis of ELR hydrogels}

The following equation was used to measure the gel hydration (Equation 1):

$$
\text { Gel hydration }(\%)=\left(\frac{W_{1}-W_{2}}{\rho_{\text {water }}}\right) * \frac{100}{V_{\text {hydrogel }}}
$$

where $W_{1}$ and $W_{2}$ refer to the weight of the swollen and lyophilized hydrogels, respectively; $\rho_{\text {water }}$ is the density of pure water, and $V_{\text {hydrogel }}$ is the volume of the hydrogel in the swollen state. 
This analysis was also conducted to have an estimated porosity of ELR hydrogels, which is related to gel hydration. Measurements were analyzed in triplicate.

\subsection{Cell Culture}

Human umbilical vein endothelial cells (HUVECs; ATCC, PCS-100-010) were cultured in EBM-2 basal medium (CC-3156, Lonza) supplemented with EGM-2 SingleQuots (CC-4176, Lonza), 10\% Fetal Bovine Serum (FBS, Lonza) and $100 \mu \mathrm{g} / \mathrm{mL}$ penicillin and streptomycin (Lonza). They were incubated at $37^{\circ} \mathrm{C}$ in a $5 \% \mathrm{CO}_{2}$ humidified atmosphere. Medium was changed twice a week and cells were used at passages between 3 and 5 for subsequent experiments.

\subsection{In vitro endothelial cell morphogenesis analysis}

A 2D cell culture system was used to evaluate the morphogenic effects of QK-ELR hydrogels. As a negative control, VEGF-depleted medium and factor-free ELR hydrogels were used. On the other hand, cell cultures used as positive controls consisted on factor-free ELR hydrogels cultured with basal medium supplemented with $10 \mathrm{ng} / \mathrm{mL}$ soluble human VEGF recombinant protein $\left(\mathrm{VEGF}_{165}\right.$, Lonza) to elicit maximum endothelial cell response, as previously reported [32]. Hydrogels were prepared in Millicell EZ slice 8-well (Thermo Scientific) by depositing $20 \mu \mathrm{L}$ of each ELR hydrogel in different wells. Subsequently, 7,000 HUVECs suspended in $200 \mu \mathrm{L}$ of medium (VEGF-depleted for QK-hydrogels and negative controls and VEGF-containing for positive controls) were seeded on top. As changes in HUVEC organization have been reported to be more apparent during the first $24 \mathrm{~h}$, plates were cultured for 2,6 and $24 \mathrm{~h}$, at $37^{\circ} \mathrm{C}$ and $5 \% \mathrm{CO}_{2}$ [35]. A complementary study to simulate the behavior of the 3D models (coaxial binary tubular scaffolds) was done. First, $5 \mu \mathrm{L}$ of the mixture of GTAR-ELR and RGD-QK-Cy5-ELR were deposited in the interior of the Millicell EZ slice well and incubated for $20 \mathrm{~min}$ at $4{ }^{\circ} \mathrm{C}$ to form the far-red fluorescent Fast-QK hydrogel. Subsequently, $10 \mu \mathrm{L}$ of the mixture of DRIR-ELR and RGD-ELR were deposited surrounding the Fast-QK hydrogel, and incubated for $20 \mathrm{~min}$ at $4{ }^{\circ} \mathrm{C}$ to form the Slow hydrogel, mimicking the scaffolds used below, but in a 2D projection. Cells were seeded as for the ELR hydrogels.

After incubation, hydrogels and scaffolds were fixed in $4 \%$ paraformaldehyde at room temperature (r.t.) for $30 \mathrm{~min}$, washed with PBS $1 \mathrm{X}$, permeabilized with $0.1 \%$ Triton $^{\mathrm{TM}} \mathrm{X}-100$ (Sigma Aldrich) and stained with PBS $1 X$ solutions containing DAPI ( $1 \mu \mathrm{g} / \mathrm{mL}$, Thermo Fisher) and Phalloidin- Alexa Fluor 488 (0.165 $\mu \mathrm{M}$, Thermo Fisher) for $30 \mathrm{~min}$ at r.t., labeling nuclei in blue and actin filaments in green, respectively. Experiments were performed in triplicate and slides were sealed with a cover glass, obtaining fluorescence images from random areas with an inverted Nikon Eclipse Ti-E epifluorescence microscope coupled to a DS-2MBWc camera using 
the NIS-Elements AR software (Nikon Corporation) and a Leica TCS SP8 LIGHTNING confocal microscope (Leica Microsystems). Cell spreading over time was analyzed by measuring their circularity $\left(4 \pi^{*}\right.$ area / perimeter $\left.{ }^{2}\right)$, as the inverse of spreading, giving a result ranging from 0 (circle, non-elongated cell) and 1 (line, completely elongated cell) [36, 37]. Circularity was calculated with customized macros in the Fiji distribution of ImageJ [38, 39]. At least 13 pictures taken from random fields of different cultures and experiments were used for quantification.

\subsection{Preparation of the 3D model ELR scaffolds and single hydrogels for the in vivo study}

Three different 3D-structured systems (coaxial binary tubular ELR scaffolds) were formed. The first one comprised the Fast hydrogel in the interior, surrounded by the Slow hydrogel, thus referred to as Slow/Fast. The other two contained the Fast-QK hydrogel in the interior, surrounded by the Slow or Slow-QK hydrogel, giving the Slow/Fast-QK and Slow-QK/Fast-QK 3D models, respectively (Table S3, Supporting Information). For their fabrication (a representative scheme of the process is depicted in Fig. S15, Supporting Information), a square-shaped silicone mold of $0.5 \mathrm{~cm}$ side and $0.6 \mathrm{~cm}$ height was used. First, a $65 \mu \mathrm{L}$ solution of the ELRs forming the Slow or Slow-QK hydrogels (external tube) was poured on the mold and incubated for crosslinking during $10 \mathrm{~min}$ at $4{ }^{\circ} \mathrm{C}$. Subsequently, $35 \mu \mathrm{L}$ of the ELRs forming the Fast or Fast-QK hydrogels were added on top and incubated for $10 \mathrm{~min}$ at $4{ }^{\circ} \mathrm{C}$, giving the internal tube. Then, two opposite sides of the scaffolds were detached from the walls of the mold. Finally, $100 \mu \mathrm{L}$ of the initial mixture (Slow or Slow-QK hydrogels) were added and incubated for $20 \mathrm{~min}$ at $4{ }^{\circ} \mathrm{C}$ to allow complete crosslinking and give the final 3D model (coaxial binary tubular scaffold). The other two sides of the scaffold were left open, thus providing access to the internal tube to cells (Fig. 1). The same mold was employed for manufacturing the single Fast, Fast-QK, Slow, SlowQK, Non-degradable and Non-degradable-QK hydrogels, with a total volume of $200 \mu \mathrm{L}$. All the procedure was performed under sterile conditions.
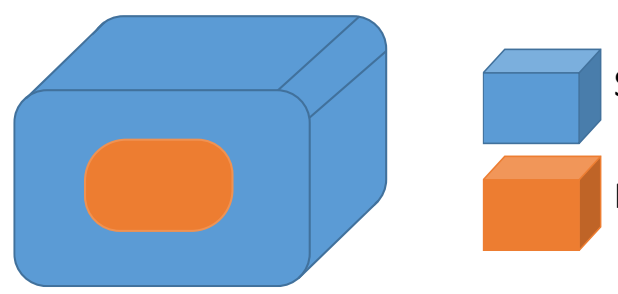

Slow or Slow-QK

Fast or Fast-QK

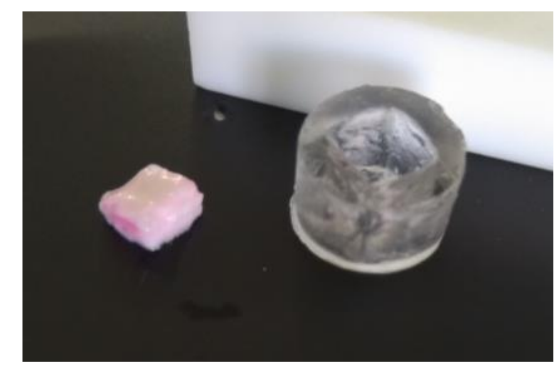

Fig. 1. Representative image of the 3D-structured systems (coaxial binary ELR tubular scaffolds), with one example of mold and final construct bearing the Fast hydrogel colored in pink. 


\subsection{Ethical approval}

Experiments including the use of animals were approved by the Animal Care and Use Committee of the University of Valladolid in accordance with the European Union Directive 2010/63/EU and the Spanish Royal Decree RD 53/2013 (resolution number 7600394).

\subsection{In vivo study}

For the in vivo study, cell-free single hydrogels and 3D models (coaxial binary tubular scaffolds) were implanted subcutaneously into female 3-month Swiss mice after anesthesia with $3 \%$ isoflurane. Specifically, a small incision was made in both sides of the spinal cord in the lumbar region using surgical scissors. Then, one hydrogel or scaffold (randomly assigned in advance) was implanted into each side (two hydrogels/scaffolds per animal) and the wound was closed with absorbable sutures. Mice were maintained on a 12:12 hours light-dark cycles and provided with water and food ad libitum. Animals were euthanized with $\mathrm{CO}_{2}$ after 1,3 and 6 weeks, collecting the hydrogels and scaffolds for their study by histology and immunohistochemistry. Three animal replicates were used for each hydrogel/scaffold and for each time point, using a total of 50 animals.

\subsection{Sample processing and histology}

After 1, 3, and 6 weeks, ELR hydrogels and 3D models (coaxial binary tubular scaffolds) were extracted from the incision site, placed in a plastic mold and covered with optimum cutting temperature (OCT) mounting medium (VWR). Then, samples were frozen by immersion in liquid nitrogen-cooled 2-methylbutane (Sigma Aldrich) and subsequently stored at $-80{ }^{\circ} \mathrm{C}$ until further processing. Once all the samples were collected, they were cut into $6-\mu \mathrm{m}$ thick sections at $-20^{\circ} \mathrm{C}$ in a cryostat (Shandon Cryotome E Cryostat, Thermo scientific).

Sample sections were stained with hematoxylin and eosin solutions (Sigma Aldrich), as previously described [40], and images were obtained with a bright-field optical microscope (Nikon Eclipse 80i) equipped with a color camera (Nikon Digital Sight DS-Fi1), using the NISElements AR software (Nikon Corporation).

\subsection{Immunohistochemistry}

Frozen slices were fixed in acetone at $-20^{\circ} \mathrm{C}$ for $2 \mathrm{~min}$ and thawed at r.t. before being rinsed three times in PBS $1 \mathrm{X}$ and permeabilized with $0.1 \%$ Triton $^{\mathrm{TM}} \mathrm{X}-100$ (Sigma-Aldrich). Subsequently, they were incubated $1 \mathrm{~h}$ at r.t. in a humidified chamber after adding $10 \%$ normal goat serum (Life Technologies). Then, they were rinsed with PBS 1X (3 times) and incubated O/N 
at $4{ }^{\circ} \mathrm{C}$ with the specific primary antibodies, all purchased from Abcam, namely anti-CD31 (endothelial cell labeling; ab28364, 1:50 dilution), anti-CCR7 (M1 labeling; ab32527, 1:100) and anti-CD163 (M2 labeling; ab182422, 1:200), diluted in 1\% normal goat serum (in PBS 1X). After three washing steps with PBS $1 \mathrm{X}$, the slices were incubated for $2 \mathrm{~h}$ at r.t. with a goat anti-rabbit IgG H\&L secondary antibody conjugated to Alexa Fluor 488 (ab150077, Abcam; 1:500) diluted in 1\% normal goat serum (in PBS 1X). Fluoroshield Mounting Medium containing DAPI (ab104139, Abcam) was used to stain cell nuclei in blue, prior to sealing with a cover glass. Sections stained with secondary antibody only were used as negative controls. Images were obtained using an inverted Nikon Eclipse Ti-E epifluorescence microscope coupled to a DS-2MBWc camera, using the NIS-Elements AR software (Nikon Corporation). Furthermore, a 3D image (Fig. $5 f$ and Video V1, Supporting Information) was obtained using a Leica TCS SP8 LIGHTNING confocal microscope (Leica Microsystems). The quantification of the overall number of cells, CD31+ cells and macrophages was conducted using a minimum of three randomly chosen areas of DAPI, CD31 and CCR7/CD163 images for each analysis, respectively. Pictures were converted to grayscale and thresholded for counting using the ImageJ software. In the case of $\mathrm{CD} 31^{+}$cells, the CD31 staining area was divided by a normalized cell area. For macrophages, the percentage was calculated by normalizing the number of $\mathrm{CCR}^{+}$or $\mathrm{CD} 163^{+}$cells with the total number of cells per area, based on DAPI staining.

\subsection{Statistical Analysis}

Data were statistically analyzed through one-way analysis of variance (ANOVA), using the HolmSidak method for post-hoc evaluation. All the experiments were performed at least in triplicate $(\mathrm{n} \geq 3)$. Results were reported as mean \pm standard deviation, accepting as statistically significant a $p$-value $<0.05 .\left({ }^{* *}\right) p<0.001$ and $\left({ }^{*}\right) p<0.05$, while n.s. $(p>0.05)$ indicates no significant differences. Origin Pro, GraphPad Prism and R (version 3.6.3) were used for graphic representation and statistical analysis.

\section{Results}

\subsection{Morphological and rheological characterization of ELR hydrogels}

SEM micrographs of Non-degradable, Non-degradable-QK, Slow, Slow-QK, Fast and Fast-QK ELR hydrogels can be found in Fig. 2a. All of them exhibited a similar porous network with a pore size of $8 \pm 2 \mu \mathrm{m}$ and a gel hydration value of $57 \pm 6 \%$ (Fig. $2 \mathrm{~b}$ ), showing a high porosity and no significant differences between the different ELR hydrogels. 
The mechanical properties of the ELR hydrogels prepared at $50 \mathrm{mg} / \mathrm{mL}$ were evaluated through rheology in the linear viscoelastic region. Accordingly, a strain-sweep experiment was performed for each hydrogel (Non-degradable, Non-degradable-QK, Slow, Slow-QK, Fast and Fast-QK) showing no dependence between the complex modulus $\left(\left|G^{*}\right|\right)$ and the strain amplitude, up to values of 7-8\% (Fig. S9-S14, Supporting Information). Fig. 2c shows the complex modulus of hydrogels at a frequency of $1 \mathrm{~Hz}, 1 \%$ strain and $37^{\circ} \mathrm{C}$. No statistically significant differences were found between the ELR hydrogels, which had a complex modulus in the range of $1220 \pm 82 \mathrm{~Pa}$.
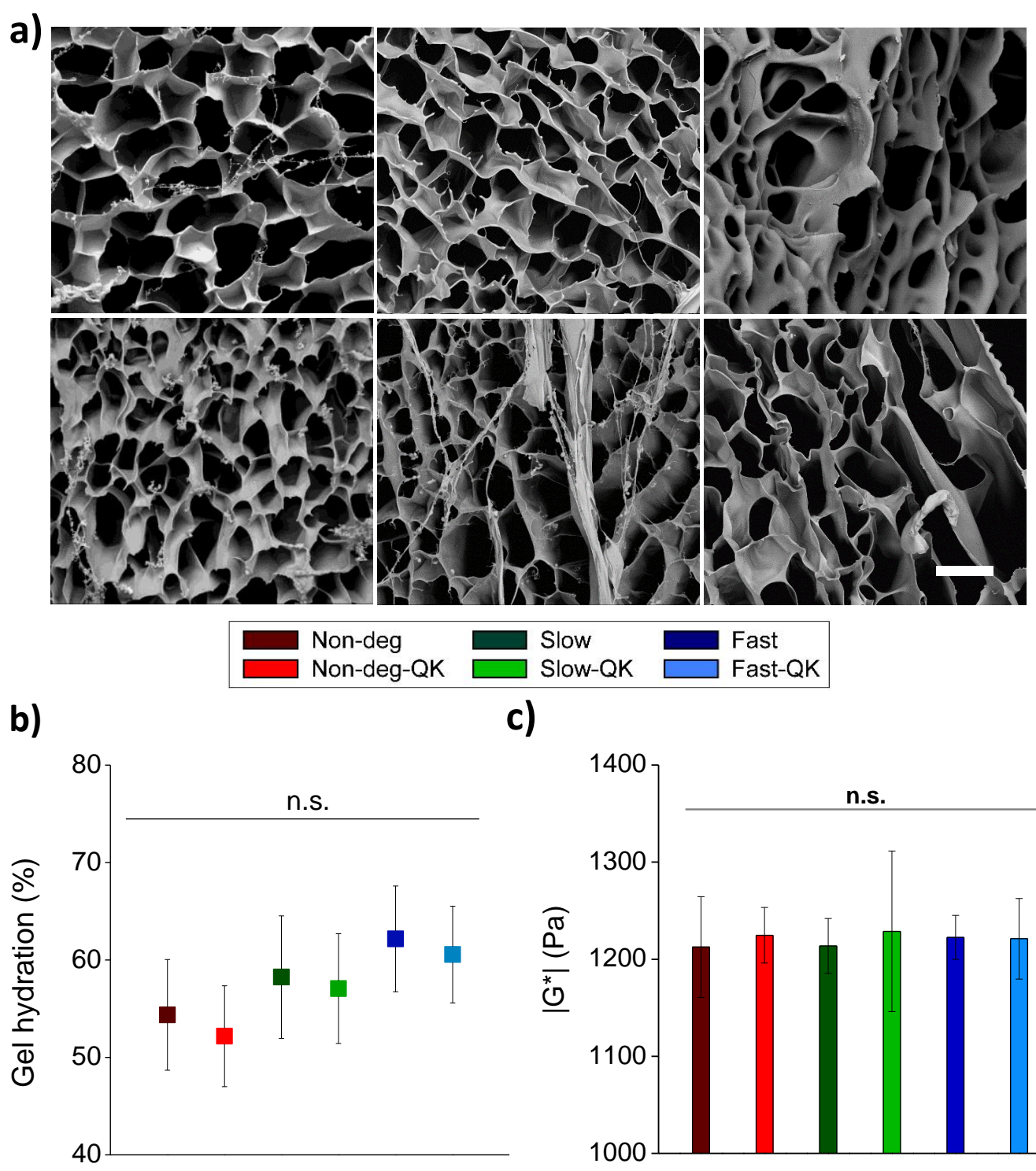

c)

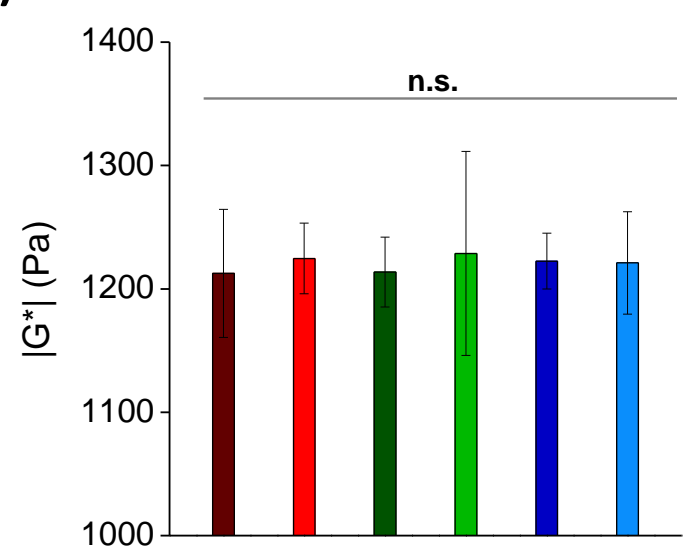

Fig. 2. Morphological and rheological characterization. (a) Representative SEM images of cryofractured, lyophilized and gold-sputtered cross sections of the Non-degradable, Slow and Fast hydrogels (from left to right on the first row), and Non-degradable-QK, Slow-QK and Fast-QK (from left to right on the second row). Scale bar $=10 \mu \mathrm{m}$. (b) Percentage of gel hydration for 
each ELR hydrogel, represented as mean \pm SD. n.s. stands for $p>0.05$. (c) Complex elastic modulus $\left|G^{*}\right|$ of the different ELR hydrogels measured at $1 \mathrm{~Hz}, 1 \%$ strain and $37^{\circ} \mathrm{C}$, represented as mean \pm SD. n.s. stands for $p>0.05$.

\subsection{In vitro morphogenesis analysis of QK-conjugated ELR hydrogels}

The pro-angiogenic activity of the tethered QK peptide was first evaluated in a $2 \mathrm{D}$ in vitro system by culturing HUVECs on top of Non-degradable, Non-degradable-QK, Slow, Slow-QK, Fast and Fast-QK ELR hydrogels, and Slow/Fast-QK coaxial binary ELR scaffolds. DAPI (nuclei) and phalloidin (actin filaments) staining (Fig. 3a for the coaxial binary ELR scaffolds and Fig. S16a, Supporting Information, for the single hydrogels) revealed signs of cell attachment after $2 \mathrm{~h}$ in all cases, due to the presence of RGD motifs [41, 42]. After $6 \mathrm{~h}$, the QK-containing hydrogels, the inner part of Slow/Fast-QK scaffolds and the positive control with VEGF V65 $_{165}$-supplemented medium showed cells with filamentous morphology tending to anastomosis (formation of network-like structures by intercellular unions). This contrasts with the factor-free hydrogels and with the outer part of Slow/Fast-QK scaffolds, where cells maintained a cobblestone morphology (Fig. 3a and Fig. S16a, Supporting Information), and this trend was even clearer after $24 \mathrm{~h}$. Furthermore, cell spreading was quantified through the measurement of cell circularity (Fig. $3 \mathrm{~b}$ and Fig. S16b, Supporting Information), which can be considered the inverse of elongation. We did not observe statistically significant differences within the different groups at $2 \mathrm{~h}$, while cell spreading was much more noticeable (i.e. circularity was lower) at 6 and $24 \mathrm{~h}$ for every case $(p<0.001)$. On the other hand, we found significant differences between the nonfunctionalized hydrogels and their QK and VEGF 165 counterparts, which showed more cell spreading both at 6 and $24 \mathrm{~h}(\mathrm{p}<0.001)$, without significant differences between them. A similar trend was observed when comparing the inner and outer parts of the scaffolds at those time points. 

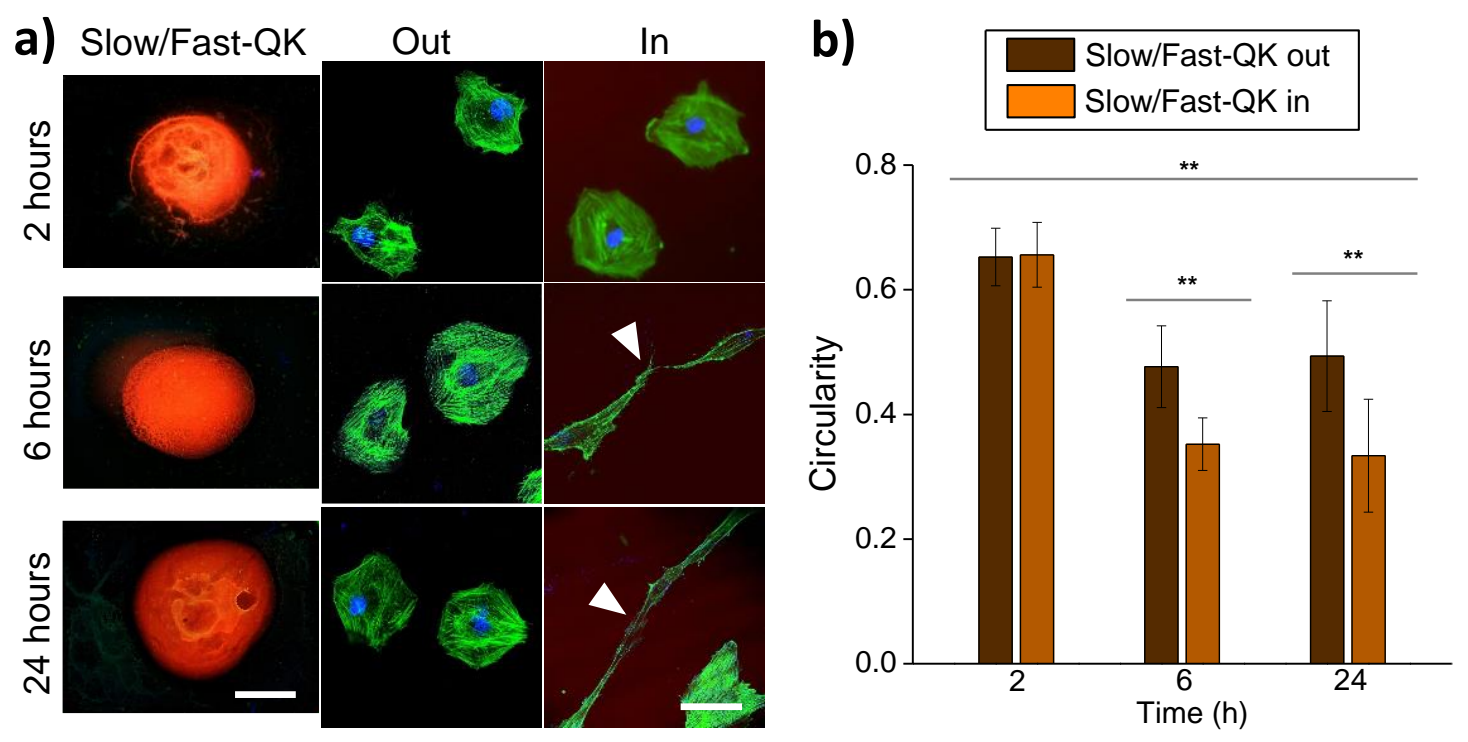

Fig. 3. Morphogenesis analysis overview. (a) Representative fluorescence images of HUVECS cultured on top of the Slow/Fast-QK hydrogels with a magnification for the inner and outer part, stained with phalloidin for actin (green) and DAPI for nuclei (blue), after 2, 6 and $24 \mathrm{~h}$. Herein, a far-red fluorescent version containing the Cy5 molecule was used for the inner part. Columns refer to the different Slow/Fast-QK and inner and outer part of Slow/Fast-QK hydrogels, while rows refer to time points. White arrows indicate cell extensions. Scale bar $=1 \mathrm{~mm}$ for Slow/FastQK and $50 \mu \mathrm{m}$ for inner and outer part of Slow/Fast-QK. (b) Circularity of cells seeded on the different parts of the Slow/Fast-QK scaffold after 2, 6 and $24 \mathrm{~h}$. Bars corresponding to the inner and outer parts are filled in a gradient of brown/orange. Data are reported as mean \pm SD. $\left({ }^{* *}\right) p<0.001$ and $(*) p<0.05$. Some statistically significant relations are not shown for clarity.

\subsection{In vivo study of single ELR hydrogels}

The formation of vasculature was evaluated in vivo by implanting Non-degradable, Nondegradable-QK, Slow, Slow-QK, Fast and Fast-QK hydrogels subcutaneously in mice. Fig. 4a shows the macroscopic appearance of the implanted hydrogels and the surrounding tissue after 6 weeks. Samples showed no harsh inflammation within the area of implantation, and all the hydrogels were surrounded by blood vessels with no presence of fibrotic tissue. Hematoxylin and eosin (H\&E) staining showed that the hydrogels kept their structure after 1 and 3 weeks in all the cases (Fig. 4b). On the other hand, Fast hydrogels were almost completely invaded after 6 weeks, contrasting with the Slow hydrogels that preserved some areas, and with Nondegradable hydrogels that maintained the complete original structure. Moreover, at this same time point, we observed a faster cleavage kinetics for the QK-containing hydrogels, being much more noticeable between the Slow and Slow-QK samples (Fig. 4b). 
Immunohistochemical staining against CD31 (a common endothelial marker) and DAPI counterstain (nuclei) were also performed to qualitatively and quantitatively analyze the number of capillaries and infiltrated cells within the hydrogels (Fig. 4c). This study revealed a statistically significant cell density increase for every group throughout the study $(p<0.001$, Fig. 4d). Particularly, host cells invaded the core of the Fast hydrogels after 3 weeks, whereas for the Slow hydrogels the inner part remained unaffected at this time point, only observing a high cell invasion after 6 weeks (Fig. 4c). Cell infiltration was much less evident in the Non-degradable hydrogels in which, even at the endpoint of the experiment, cells appeared preferentially distributed surrounding the hydrogel, indicating a much slower cell colonization rate (Fig. 4c). Furthermore, statistically relevant differences were found between Fast and Fast-QK hydrogels in terms of cell density at 1 and 3 weeks ( $<0.001$ and $p<0.05$, respectively). Similarly, significant differences were observed between Slow and Slow-QK hydrogels after 6 weeks $(p<$ $0.05)$, thus confirming the relation between the presence of the QK peptide and a higher cell infiltration rate (Fig. 4d). Furthermore, anti-CD31 immunostaining revealed a high density of endothelial cells forming capillaries in Fast, Fast-QK, Slow and Slow-QK hydrogels at 6 weeks post-implantation, with no vascularization observed in Non-degradable hydrogels at any time point. In addition, we found differences in the number of capillaries within Fast and Slow hydrogels $(p<0.001)$, and between the QK-containing hydrogels and their factor-free counterparts ( $p<0.001$, Fig. 4e). A representative 3D image of a random capillary within the Fast hydrogel after 6 weeks is shown in Fig. $4 \mathrm{f}$ (see also Video V1, Supporting Information). 

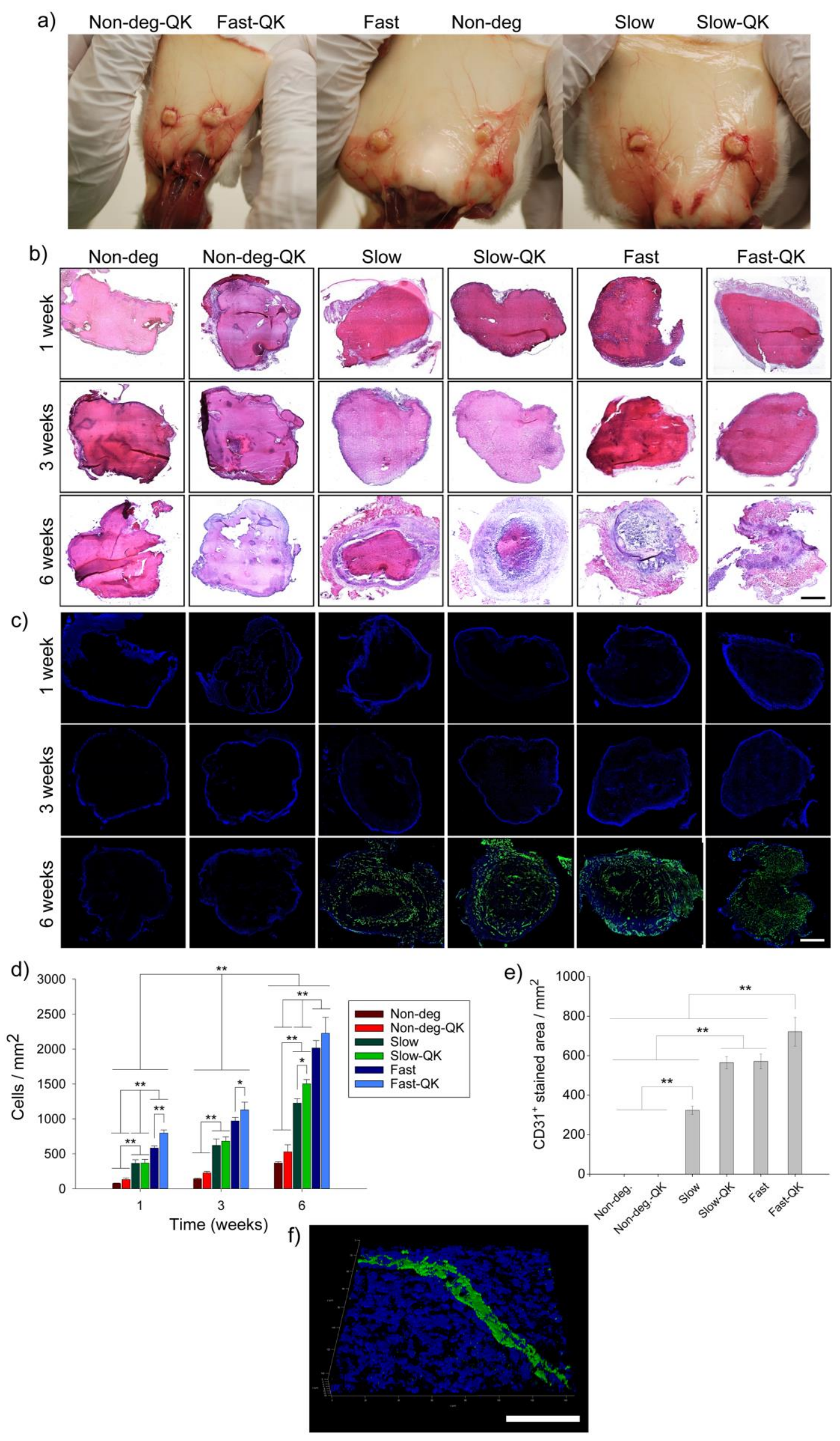
Fig. 4. In vivo single ELR hydrogels analysis. (a) Representative images of the subcutaneously implanted hydrogels in mice after 6 weeks. (b) Representative hematoxylin and eosin (H\&E)stained hydrogel sections. Columns correspond to the different ELR hydrogels, while rows indicate time points. Scale bar $=2 \mathrm{~mm}$. (c) Representative images of DAPI and CD31-stained hydrogel sections, where the nuclei of cells appear in blue and endothelial cells in green. Columns correspond to the different ELR hydrogels while rows indicate time points. Scale bar = $2 \mathrm{~mm}$. (d) Quantification of cell invasion (number of cells per $\mathrm{mm}^{2}$ ) within the ELR hydrogels after 1, 3 and 6 weeks post-implantation. Results for Non-degradable hydrogels are shown in red, Slow in green and Fast in blue. QK-containing hydrogels are represented with a brighter hue of the corresponding color. (e) $\mathrm{CD} 31^{+}$stained area per $\mathrm{mm}^{2}$ within the ELR hydrogels after 6 weeks post-implantation. (d) and (e) Data are reported as mean \pm SD. $\left({ }^{* *}\right) p<0.001$ and $\left({ }^{*}\right) p<$ 0.05. The differences highlighted for different time points refer to the comparison between samples of the same group, although other differences might be commented in the text. Some statistically significant comparisons are not shown for clarity. (f) Representative 3D image of a random capillary within the Fast hydrogel after 6 weeks.

\subsection{In vivo study of 3D model ELR scaffolds}

The preferential spatiotemporal formation of micro-vessels in the pre-designed 3DSlow/Fast, Slow/Fast-QK and Slow-QK/Fast-QK models (coaxial binary tubular scaffolds) was evaluated in vivo through their subcutaneous implantation in mice. Specifically, the Slow/Fast scaffold consists of an external Slow hydrogel and an internal Fast hydrogel. On the other hand, the Slow/Fast-QK and Slow-QK/Fast-QK scaffolds were further designed displaying the QK peptide in the inside only or in both parts, respectively, while conserving the proteolytic sensitivity of the Slow/Fast scaffold. The macroscopic morphology of the scaffolds was similar for all the groups and samples, as confirmed by simple visualization, showing high reproducibility, and we always made sure that the coaxial tubular structure was obtained. H\&E-stained slides (Fig. 5a) show that the 3D model structure was preserved after 1 week. In contrast, the outer tube remained intact after 3 weeks, whereas some signs of degradation were observed for the inner tube of all the types of scaffolds. The degradation of both tubes continued after 6 weeks, leading to the complete degradation of the Slow-QK/Fast-QK scaffold, while some remnants of the outer part were found for the other two groups.

Anti-CD31 immunofluorescence staining and DAPI counterstaining allowed the qualitative and quantitative evaluation of cell invasion and the formation of new capillaries within the 3D models (Fig. 5b). After 1 week, a low cell infiltration was found for the outer tube, whereas 
several isolated cells were appreciated for the inner tube (Fig. 5b). The samples retrieved after 3 weeks evidenced an increase in cell infiltration in the inner tube, together with a partially invaded outer tube, and this trend continued after 6 weeks (Fig. 5b). Indeed, the inner and outer tube displayed significant differences in cell invasion within all the 3D models at every time point (Fig. $5 c, p<0.001$ ). Moreover, regarding the role of the QK peptide on the promotion of cell invasion, we found statistically significant differences in the number of cells $/ \mathrm{mm}^{2}$ between the outer tube (Slow hydrogel) of the Slow-QK/Fast-QK and of both the Slow/Fast and Slow/Fast-QK scaffolds after 6 weeks $(p<0.05)$. In addition, this QK-related response was also observed within the inner tube displaying faster cleavage kinetics (Fast hydrogel), giving differences between the Slow/Fast and both the Slow/Fast-QK and Slow-QK/Fast-QK scaffolds after 1 week $(p<0.001)$ and 3 weeks $(p<0.05)$ (Fig. $5 c)$. These results were in agreement with those found for the single hydrogels (Fig. 4).

Furthermore, CD31 immunostaining showed that there was no sign of CD31-positive cells after 1 week, whereas we observed a high density of green areas for the inner tube and dispersed green stained cells for the outer one after 3 weeks (Fig. 5b). This trend continued for the Slow/Fast and Slow/Fast-QK scaffolds, thus evidencing a proportional intensification of this pattern after 6 weeks. On the other hand, dense capillary networks were found colonizing both the inner and outer tube of the Slow-QK/Fast-QK scaffold at this time point (Fig. 5b). Indeed, we found significant differences between this and the other two scaffolds in terms of number of capillaries within the outer tube after 6 weeks $(p<0.001)$, hence confirming the pro-angiogenic effect of the conjugated QK peptide (Fig. 5d). 

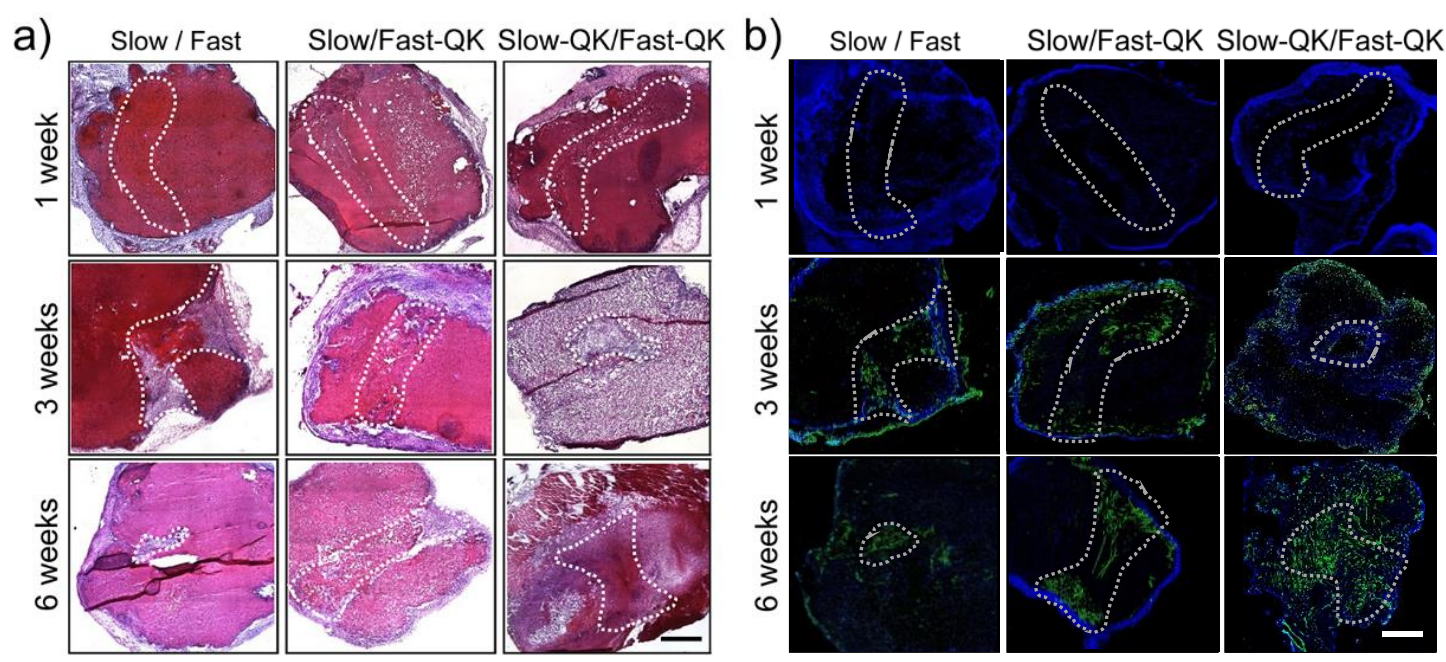

Slow/Fast out Slow/Fast in Slow/Fast-QK out Slow/Fast-QK in

Slow-QK/Fast-QK out Slow-QK/Fast-QK in

c)

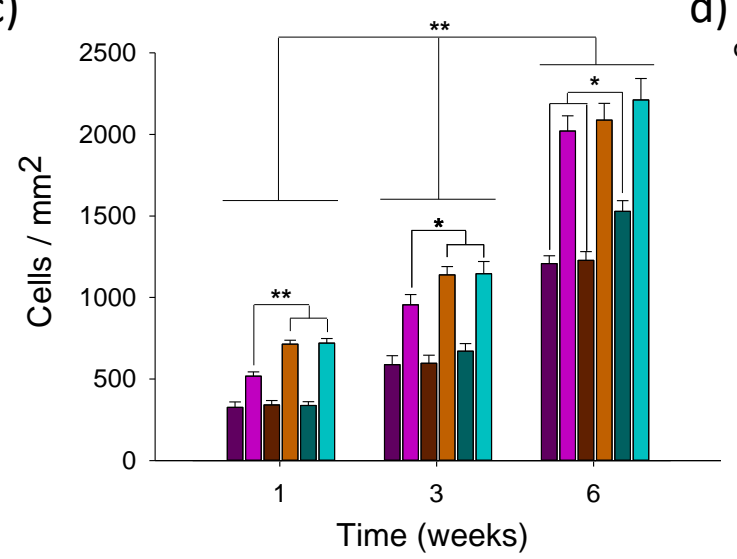

d)

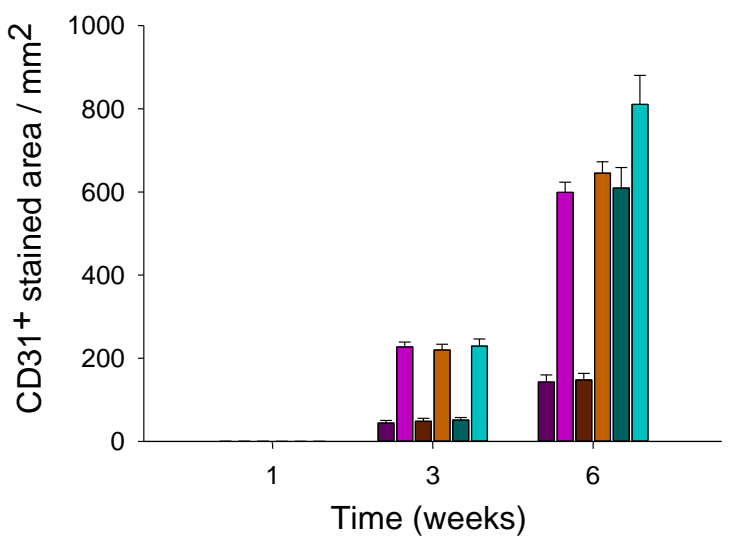

Fig. 5. In vivo 3D model ELR scaffolds analysis (a) Representative images of the H\&E stained scaffolds, where the inner tube is surrounded by a white dotted line. Columns correspond to the type of 3D model (coaxial binary tubular scaffold), whereas rows correspond to the time points. Scale bar = $2 \mathrm{~mm}$. (b) Representative images of the DAPI (nuclei, blue) and CD31 (endothelial cells, green) stained scaffolds, where the inner tube is surrounded by a white dotted line. Columns correspond to the type of 3D model (coaxial binary tubular scaffold), while rows correspond to the time points. Scale bar $=2 \mathrm{~mm}$. (c) Number of cells per $\mathrm{mm}^{2}$ for the inner and outer tubes that conform the 3D models (coaxial binary tubular scaffolds) at 1, 3 and 6 weeks post-implantation. (d) $\mathrm{CD} 31^{+}$stained area per $\mathrm{mm}^{2}$ for the inner and outer tubes that conform the 3D models (coaxial binary tubular scaffolds) at 1, 3 and 6 weeks post-implantation. (c) and (d) Results for Slow/Fast are shown in red, Slow/Fast-QK in green and Slow-QK/Fast-QK in blue. Inner tubes are represented with a brighter hue of the corresponding color. Data are reported as mean \pm SD. $\left({ }^{* *}\right) p<0.001$ and $(*) p<0.05$. The differences highlighted for different time points refer to the comparison between samples of the same group, although other differences 
might be commented in the text. Some statistically significant comparisons are not shown for clarity. Differences between the inner and outer membranes of all the scaffolds are not highlighted for clarity, but they were significant at every time point $(p<0.001)$.

\subsection{Evaluation of the macrophage response to single ELR hydrogels}

The in vivo immunological response of the ELR hydrogels was evaluated via immunohistochemical CCR7 and CD163 staining of the samples, thereby labeling proinflammatory (M1) and pro-healing (M2) macrophages, respectively. This analysis revealed a higher density of both types of macrophages in Non-degradable and Non-degradable-QK hydrogels when compared to Slow, Slow-QK, Fast and Fast-QK hydrogels after 1 and 3 weeks (Fig. 6a-b and Fig. S17a-b, Supporting Information). After 6 weeks, the inflammatory response decreased in all of them, which resulted in a residual number of both types of macrophages, with no statistically significant differences between the different hydrogels (Fig. S17a-b). The presence of the QK peptide revealed no influence on macrophage type and amount, suggesting no immune response associated to its presence. The $M 1 / M 2$ ratio reported a slight predominance in M1 macrophages, with a highest value of $1.27 \pm 0.02$ for Non-degradable, Nondegradable-QK, Slow and Slow-QK hydrogels, compared to $1.08 \pm 0.01$ of Fast and Fast-QK hydrogels after 1 week (Fig. 6c). This ratio was similar after 3 weeks for Non-degradable and Non-degradable-QK hydrogels $(1.25 \pm 0.02)$, whereas a statistically significant decrease was found for Slow and Slow-QK hydrogels $(1.16 \pm 0.01, p<0.001)$ and Fast and Fast-QK hydrogels $(1.04 \pm 0.01, p<0.05)$, in comparison to the previous time point. This progression continued over time, resulting in, approximately, a 1:1 ratio for all the hydrogels after 6 weeks. 


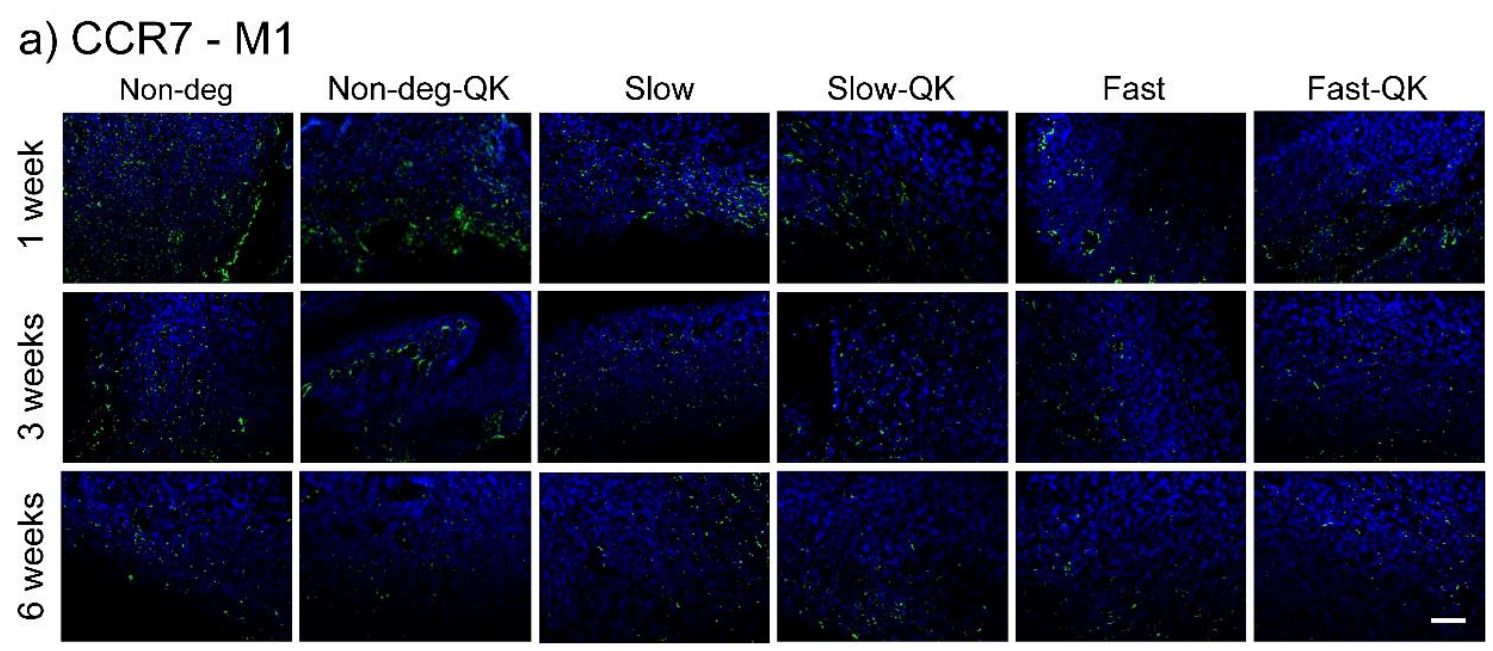

b) CD163 - M2
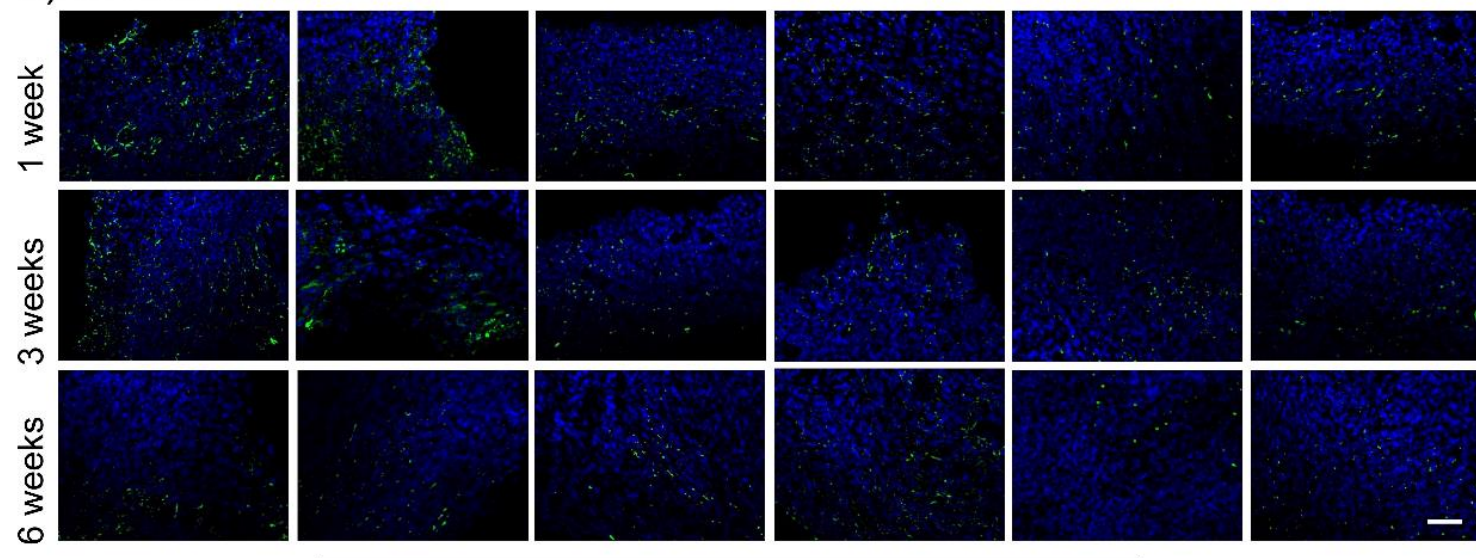

Non-deg

Slow

Slow-QK

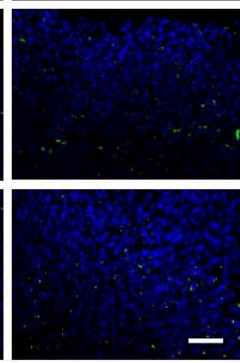

Non-deg-QK

c)

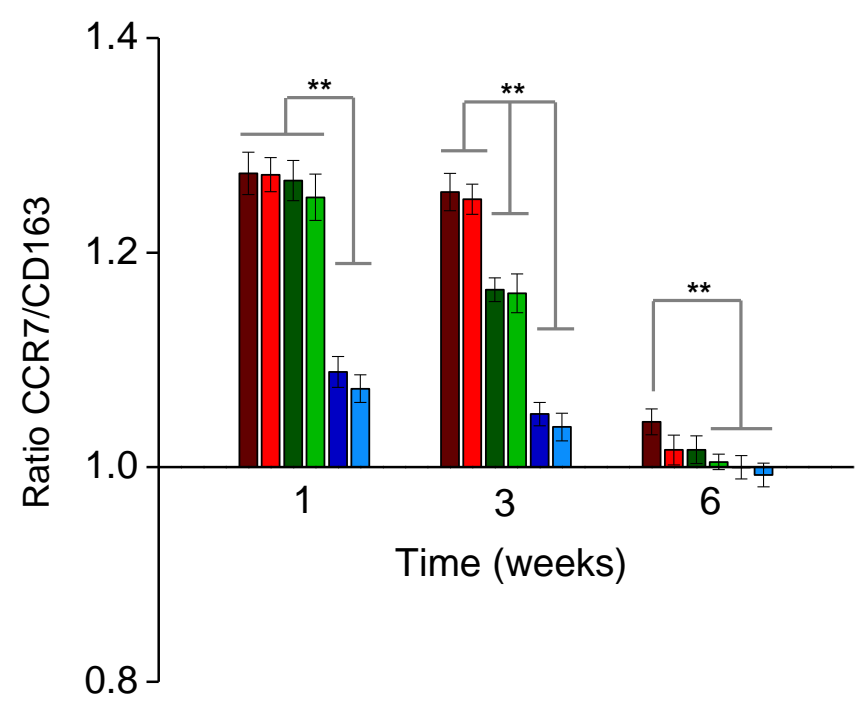

Fig. 6. In vivo macrophage response analysis (a) Representative images of the DAPI (nuclei, blue)and CCR7 (M1 macrophages, green)-stained single ELR hydrogels. Columns correspond to the different hydrogels, while rows correspond to time points. Scale bar $=50 \mu \mathrm{m}$ (b) Representative 
images of the DAPI (nuclei, blue)- and CD163 (M2 macrophages, green)-stained scaffolds. Columns correspond to the different hydrogels, while rows correspond to time points. Scale bar $=50 \mu \mathrm{m}$. (c) CCR7/CD163 ratio (M1/M2, respectively) for the different hydrogels at the time points evaluated. Data are reported as mean \pm SD. $\left({ }^{* *}\right) p<0.001$ and $\left({ }^{*}\right) p<0.05$. Differences between time points are not shown for clarity, but they are indicated in the text.

\section{Discussion}

The challenge of controlled angiogenesis in scaffolds has been largely aimed in TERM, mainly by using synthetic and ECM-like hydrogels bearing proteolytic cues and covalently tethered biochemical signals $[6,8-10,43-47]$. In this work, we have demonstrated that 3D models consisting of coaxial binary ELR tubular scaffolds enabled the spatiotemporal control of angiogenesis, showing the formation of micro-vessels in a pre-defined direction, ahead of the overall natural cell infiltration process, upon implantation in vivo. Our approach involved the use of proteolytic sequences with different cleavage kinetics, cell-adhesive RGD domains and the pro-angiogenic QK peptide.

The ELR hydrogels used for this study were first physically characterized for their applicability as soft tissue substitutes. SEM results allowed to identify the porous microstructure, while rheology measurements showed the soft stiffness exhibited by the ELR hydrogels, comparable with biological scaffolds suitable for TERM purposes [19, 31, 48, 49]. In vitro studies confirmed the pro-angiogenic activity of the QK-conjugated hydrogels on HUVEC morphology, in agreement with other ELR, collagen and poly(ethylene glycol) diacrylate (PEGDA) QK-bearing hydrogels, and similar to the soluble VEGF $\mathrm{VE5}_{165}$, used herein as positive control [11, 50-52]. Subsequently, the subcutaneous implantation of ELR hydrogels in a mouse model revealed a high cell infiltration for Fast and Fast-QK hydrogels, and to a minor extent for Slow and Slow-QK hydrogels, which contrasts with the much limited cell invasion observed for Non-degradable and Non-degradable-QK controls even at the latest time point evaluated. Results further evidenced the direct relationship existing between the QK peptide and cell infiltration rate, amplifying the effect of the proteolytic disruption of the hydrogels for Fast and Slow groups after 6 weeks, but not having an effect for the Non-degradable hydrogel. In addition, anti-CD31 immunostaining reported a larger number of newly formed capillaries in Fast and Slow ELR hydrogels after 6 weeks, which was intensified in the presence of the QK-peptide. Thus, this manifests the synergy between proteolytic sequences (totally necessary) and the QK peptide in the promotion of vascularization, as well as in cell infiltration and tissue integration. In the absence of growth factors, Sokic et al. have already demonstrated the impact of the cleavage kinetics in the neovascularization process in vivo, comparing PEG hydrogels with varying MMP-sensitive 
sequences concentrations [45]. Flora et al. have also reported comparable observations on ELR hydrogels bearing sequences (similar to the ones used in this study) with different proteolytic kinetics towards the uPA protease, showing the importance of tuning the sensitivity to proteases to modulate vascularization in vivo [31]. As regards biochemical signaling, the attachment of the QK peptide has also shown promising results. In an in vivo study, Leslie-Barbick et al. demonstrated an improvement in the promotion of angiogenesis for collagenase-degradable PEG-QK hydrogel bearing RGD sequences when compared to hydrogels with embedded growth factors, and PEG-VEGF or PEG-RGDS hydrogels [46]. Similarly, Flora et al. have described an increase in the formation of capillaries in vivo with ELR-QK hydrogels containing RGD and REDV cell adhesion sequences, in comparison with factor-free ELR hydrogels [31], also in agreement with a RGD- and REDV-bioactivated ELR hydrogel, as reported by Staubli et al [53]. Although these scaffolds promoted angiogenesis and induced a faster formation of micro-vessels, random orientations were observed for the newly formed vasculature evidencing the inability of these systems to recapitulate the complexity of native tissues $[43,47,54]$.

Based on the single ELR hydrogel results, we fabricated 3D models consisting of coaxial binary ELR tubular scaffolds to study their behavior when implanted in vivo. We used different combinations of tubes, including the QK peptide in none, both or in the internal tube only, but always using the Slow hydrogel for the outer tube and the Fast hydrogel for the internal tube. Their subcutaneous implantation in mice reproduced the response exhibited by the single hydrogels, thus finding signs of degradation and cell infiltration after 3 weeks in the inner tube containing the Fast or Fast-QK hydrogel, with initial formation of capillaries. After 6 weeks, the scaffolds lacking QK peptide in the outer tube still showed anisotropic vascularization and cell infiltration focused on the internal part, whereas the scaffolds containing the QK peptide in both parts reported an advanced cell invasion and scaffold disintegration, with a large number of capillaries dispersed within almost the whole scaffold. In terms of spatiotemporal direction of angiogenesis, Yuen et al. have described the use of a microporous biodegradable poly(lactic-coglycolic) (PLG) copolymer to form a three-layered cylindrical scaffold embedding vascular endothelial growth factor (VEGF) in the central layer and anti-VEGF factor in the surrounding layers [43]. This arrangement created a spatially restricted angiogenic region that was maintained for 3 weeks in vivo. However, the construct exhibited an initial burst release of VEGF and anti-VEGF factor during the first 3 days (of $60 \%$ and $75 \%$, respectively), which can potentially lead to uncontrolled neovascularization or tumor growth. Contrarily, in our work we have combined dynamic mechanobiological cues in ELR scaffolds that can resemble and adapt to the native ECM, while guiding cell invasion and angiogenesis. 
As regards immunogenicity, protease-sensitive ELR hydrogels showed a reduced initial inflammatory response, in comparison to Non-degradable ones, and this was accompanied by a decrease in macrophage number during time in every case. This indicated an inverse correlation between cleavage kinetics and macrophage content, with no differences upon conjugation with the QK-peptide. In addition, the M1/M2 ratio confirmed an equilibrated presence of both types of macrophages with a slight tendency towards pro-healing macrophages (M2) over time, and without signs of chronic inflammation or fibrotic tissue formation, in agreement with previously described protease-sensitive ELR hydrogels $[20,25]$. Thus, in terms of macrophage-related immunogenicity, these hydrogels and scaffolds can be safely used for TERM applications that require the formation of a complex vascular structure.

\section{Conclusions}

Herein, the merge of two ELRs including proteolytic sequences with different cleavage kinetics and a VEGF-mimetic tethered peptide (QK) into bioactive 3D models (coaxial binary tubular scaffolds) allowed guiding angiogenesis in a pre-designed spatiotemporal dimension. The synergistic effect of both factors towards cell infiltration and subsequent vascularization was confirmed. Furthermore, the protease-sensitive ELR hydrogels induced a mild macrophage response, which shifted towards $\mathrm{M} 2$ and resolved over time, supporting the potential integration and adaptability of the implant within the host tissue. Therefore, we have shown that cell colonization and vascularization can be spatiotemporally programed selecting the bioactivities and the cleavage kinetics engineered in complex ELR scaffolds. This work presents relevant insights in the development of a 3D model that may find uses in a wide number of tissue engineering applications where the precise control of angiogenesis plays an important role, offering at the same time a biocompatible and resorbable scaffold.

\section{Acknowledgements}

The authors are grateful for the funding from the Spanish Government (MAT2016-78903-R, RTI2018-096320-B-C22, FPU16/04015), Junta de Castilla y León (VA317P18), Interreg V España Portugal POCTEP (0624_2IQBIONEURO_6_E), Centro en Red de Medicina Regenerativa y Terapia Celular de Castilla y León and proyecto UVA06 de la convocatoria Infraestructuras en Red de Castilla y León 2019 de la Junta de Castilla y León. Authors also would like to thank Rocío García for the support in the bioproduction of the different ELRs used in this work.

7. Conflicts of interest

Authors declare no conflicts of interest. 


\section{ORCID iDs}

Fernando González-Pérez https://orcid.org/0000-0003-3170-3035

Arturo Ibáñez-Fonseca https://orcid.org/0000-0002-2223-5041

Matilde Alonso https://orcid.org/0000-0002-6853-8427

José Carlos Rodríguez-Cabello https://orcid.org/0000-0002-3438-858X

9. References

[1] J.L. West, J.J. Moon, Vascularization of Engineered Tissues: Approaches to Promote Angiogenesis in Biomaterials, Curr. Top. Med. Chem. 8 (n.d.) 300-310.

[2] J. Rouwkema, A. Khademhosseini, Vascularization and Angiogenesis in Tissue Engineering: Beyond Creating Static Networks, Trends Biotechnol. 34 (2016) 733-745.

[3] J.E. Rundhaug, Matrix metalloproteinases and angiogenesis, J. Cell. Mol. Med. 9 (2005) 267-285.

[4] V. Mastrullo, W. Cathery, E. Velliou, P. Madeddu, P. Campagnolo, Angiogenesis in tissue engineering: as nature intended?, Front. Bioeng. Biotechnol. 8 (2020) 188.

[5] R.J. Kant, K.L.K. Coulombe, Integrated approaches to spatiotemporally directing angiogenesis in host and engineered tissues, Acta Biomater. 69 (2018) 42-62.

[6] J. Leijten, J. Seo, K. Yue, G. Trujillo-de Santiago, A. Tamayol, G.U. Ruiz-Esparza, S.R. Shin, R. Sharifi, I. Noshadi, M.M. Álvarez, Y.S. Zhang, A. Khademhosseini, Spatially and temporally controlled hydrogels for tissue engineering, Mater. Sci. Eng. R Reports. 119 (2017) 1-35.

[7] M. Lovett, K. Lee, A. Edwards, D.L. Kaplan, Vascularization Strategies for Tissue Engineering, Tissue Eng. Part B Rev. 15 (2009) 353-370.

[8] L.L.Y. Chiu, M. Radisic, Scaffolds with covalently immobilized VEGF and Angiopoietin-1 for vascularization of engineered tissues, Biomaterials. 31 (2010) 226-241.

[9] P.R. Kuhl, L.G. Griffith-Cima, Tethered epidermal growth factor as a paradigm for growth factor-induced stimulation from the solid phase, Nat. Med. 2 (1996) 1022-1027.

[10] L.L.Y. Chiu, R.D. Weisel, R. Li, M. Radisic, Defining conditions for covalent immobilization of angiogenic growth factors onto scaffolds for tissue engineering, J. Tissue Eng. Regen. Med. 5 (2011) 69-84.

[11] L. Cai, C.B. Dinh, S.C. Heilshorn, One-pot synthesis of elastin-like polypeptide hydrogels with grafted VEGF-mimetic peptides, Biomater. Sci. 2 (2014) 757-765.

[12] A. Girotti, D. Orbanic, A. Ibáñez-Fonseca, C. Gonzalez-Obeso, J.C. Rodríguez-Cabello, Recombinant Technology in the Development of Materials and Systems for Soft-Tissue Repair, Adv. Healthc. Mater. 4 (2015) 2423-2455.

[13] D.W. Urry, T.M. Parker, M.C. Reid, D.C. Gowda, Biocompatibility of the Bioelastic Materials, Poly(GVGVP) and Its $\gamma$-Irradiation Cross-Linked Matrix: Summary of Generic Biological Test Results, J. Bioact. Compat. Polym. 6 (1991) 263-282.

[14] D.W. Urry, C.H. Luan, T.M. Parker, D.C. Gowda, K.U. Prasad, M.C. Reid, A. Safavy, Temperature of polypeptide inverse temperature transition depends on mean residue 
hydrophobicity, J. Am. Chem. Soc. 113 (1991) 4346-4348.

[15] D.T. McPherson, J. Xu, D.W. Urry, Product Purification by Reversible Phase Transition FollowingEscherichia coliExpression of Genes Encoding up to 251 Repeats of the Elastomeric Pentapeptide GVGVP, Protein Expr. Purif. 7 (1996) 51-57.

[16] H.C. Kolb, M.G. Finn, K.B. Sharpless, Click chemistry: diverse chemical function from a few good reactions, Angew. Chemie Int. Ed. 40 (2001) 2004-2021.

[17] A.M. Testera, A. Girotti, I.G. de Torre, L. Quintanilla, M. Santos, M. Alonso, J.C. RodríguezCabello, Biocompatible elastin-like click gels: design, synthesis and characterization, J. Mater. Sci. Mater. Med. 26 (2015) 105.

[18] N.J. Agard, J.A. Prescher, C.R. Bertozzi, A strain-promoted [3+ 2] azide- alkyne cycloaddition for covalent modification of biomolecules in living systems, J. Am. Chem. Soc. 126 (2004) 15046-15047.

[19] I.G. de Torre, M. Santos, L. Quintanilla, A. Testera, M. Alonso, J.C.R. Cabello, Elastin-like recombinamer catalyst-free click gels: characterization of poroelastic and intrinsic viscoelastic properties, Acta Biomater. 10 (2014) 2495-2505.

[20] A. Ibáñez-Fonseca, T.L. Ramos, I. Gonzalez de Torre, L.I. Sánchez-Abarca, S. Muntión, F.J. Arias, M.C. Del Cañizo, M. Alonso, F. Sánchez-Guijo, J.C. Rodríguez-Cabello, Biocompatibility of two model elastin-like recombinamer-based hydrogels formed through physical or chemical cross-linking for various applications in tissue engineering and regenerative medicine, J. Tissue Eng. Regen. Med. 12 (2018) e1450-e1460.

[21] A. Ibáñez-Fonseca, T. Flora, S. Acosta, J.C. Rodríguez-Cabello, Trends in the design and use of elastin-like recombinamers as biomaterials, Matrix Biol. 84 (2019) 111-126.

[22] D.J. Coletta, A. Ibáñez-Fonseca, L.R. Missana, M. V Jammal, E.J. Vitelli, M. Aimone, F. Zabalza, J.P.M. Issa, M. Alonso, J.C. Rodríguez-Cabello, Bone regeneration mediated by a bioactive and biodegradable extracellular matrix-like hydrogel based on elastin-like recombinamers, Tissue Eng. Part A. 23 (2017) 1361-1371.

[23] D. Pescador, A. Ibáñez-Fonseca, F. Sánchez-Guijo, J.G. Briñón, F.J. Arias, S. Muntión, C. Hernández, A. Girotti, M. Alonso, M.C. Del Cañizo, Regeneration of hyaline cartilage promoted by xenogeneic mesenchymal stromal cells embedded within elastin-like recombinamer-based bioactive hydrogels, J. Mater. Sci. Mater. Med. 28 (2017) 1-11.

[24] F. Cipriani, B. Ariño Palao, I. Gonzalez De Torre, A. Vega Castrillo, H.J. Aguado Hernández, M. Alonso Rodrigo, A.J. Àlvarez Barcia, A. Sanchez, V. García Diaz, M. Lopez Peña, J.C. Rodriguez-Cabello, An elastin-like recombinamer-based bioactive hydrogel embedded with mesenchymal stromal cells as an injectable scaffold for osteochondral repair, Regen. Biomater. 6 (2019) 335-347.

[25] A. Ibáñez-Fonseca, S. Santiago Maniega, D. Gorbenko del Blanco, B. Catalán Bernardos, A. Vega Castrillo, Á.J. Álvarez Barcia, M. Alonso, H.J. Aguado, J.C. Rodríguez-Cabello, Elastin-Like Recombinamer Hydrogels for Improved Skeletal Muscle Healing Through Modulation of Macrophage Polarization, Front. Bioeng. Biotechnol. 8 (2020) 413.

[26] F. Chen, P. Le, G.M. Fernandes-Cunha, S.C. Heilshorn, D. Myung, Bio-orthogonally crosslinked hyaluronate-collagen hydrogel for suture-free corneal defect repair, Biomaterials. 255 (2020) 120176.

[27] A. Fernández-Colino, F. Wolf, S. Rütten, T. Schmitz-Rode, J.C. Rodríguez-Cabello, S. Jockenhoevel, P. Mela, Small Caliber Compliant Vascular Grafts Based on Elastin-Like Recombinamers for in situ Tissue Engineering , Front. Bioeng. Biotechnol. . 7 (2019) 340. 
[28] A. Fernández-Colino, F. Wolf, R. Moreira, S. Rütten, T. Schmitz-Rode, J.C. RodríguezCabello, S. Jockenhoevel, P. Mela, Layer-by-layer biofabrication of coronary covered stents with clickable elastin-like recombinamers, Eur. Polym. J. 121 (2019) 109334.

[29] P. Contessotto, D. Orbanić, M. Da Costa, C. Jin, P. Owens, S. Chantepie, C. Chinello, J. Newell, F. Magni, D. Papy-Garcia, Elastin-like recombinamers-based hydrogel modulates post-ischemic remodeling in a non-transmural myocardial infarction in sheep, Sci. Transl. Med. 13 (2021).

[30] T. Flora, I.G. de Torre, M. Alonso, J.C. Rodríguez-Cabello, Tethering QK peptide to enhance angiogenesis in elastin-like recombinamer (ELR) hydrogels, J. Mater. Sci. Mater. Med. 30 (2019) 1-12.

[31] T. Flora, I.G. De Torre, M. Alonso, J.C. Rodríguez-Cabello, Use of proteolytic sequences with different cleavage kinetics as a way to generate hydrogels with preprogrammed cellinfiltration patterns imparted over their given 3D spatial structure, Biofabrication. 11 (2019) 35008.

[32] L.D. D'Andrea, G. Iaccarino, R. Fattorusso, D. Sorriento, C. Carannante, D. Capasso, B. Trimarco, C. Pedone, Targeting angiogenesis: structural characterization and biological properties of a de novo engineered VEGF mimicking peptide, Proc. Natl. Acad. Sci. 102 (2005) 14215-14220.

[33] A. Girotti, J. Reguera, J.C. Rodríguez-Cabello, F.J. Arias, M. Alonso, A.M. Testera, Design and bioproduction of a recombinant multi (bio) functional elastin-like protein polymer containing cell adhesion sequences for tissue engineering purposes, J. Mater. Sci. Mater. Med. 15 (2004) 479-484.

[34] C.T. Rueden, J. Schindelin, M.C. Hiner, B.E. DeZonia, A.E. Walter, E.T. Arena, K.W. Eliceiri, Image 2: ImageJ for the next generation of scientific image data, BMC Bioinformatics. 18 (2017) 1-26.

[35] J.T. Koepsel, E.H. Nguyen, W.L. Murphy, Differential effects of a soluble or immobilized VEGFR-binding peptide, Integr. Biol. 4 (2012) 914-924.

[36] C.R. Wittmer, J.A. Phelps, W.M. Saltzman, P.R. Van Tassel, Fibronectin terminated multilayer films: protein adsorption and cell attachment studies, Biomaterials. 28 (2007) $851-860$.

[37] C.E. Vorwald, T. Gonzalez-Fernandez, S. Joshee, P. Sikorski, J.K. Leach, Tunable fibrinalginate interpenetrating network hydrogels to support cell spreading and network formation, Acta Biomater. 108 (2020) 142-152.

[38] J. Schindelin, I. Arganda-Carreras, E. Frise, V. Kaynig, M. Longair, T. Pietzsch, S. Preibisch, C. Rueden, S. Saalfeld, B. Schmid, Fiji: an open-source platform for biological-image analysis, Nat. Methods. 9 (2012) 676-682.

[39] C.A. Schneider, W.S. Rasband, K.W. Eliceiri, NIH Image to ImageJ: 25 years of image analysis, Nat. Methods. 9 (2012) 671-675.

[40] A.H. Fischer, K.A. Jacobson, J. Rose, R. Zeller, Hematoxylin and Eosin Staining of Tissue and Cell Sections, Cold Spring Harb. Protoc. . 2008 (2008) pdb.prot4986.

[41] E. Ruoslahti, M.D. Pierschbacher, New perspectives in cell adhesion: RGD and integrins, Science (80-. ). 238 (1987) 491 LP - 497.

[42] M. Pierna, M. Santos, F.J. Arias, M. Alonso, J.C. Rodríguez-Cabello, Efficient cell and cellsheet harvesting based on smart surfaces coated with a multifunctional and selforganizing elastin-like recombinamer, Biomacromolecules. 14 (2013) 1893-1903. 
[43] W.W. Yuen, N.R. Du, C.H. Chan, E.A. Silva, D.J. Mooney, Mimicking nature by codelivery of stimulant and inhibitor to create temporally stable and spatially restricted angiogenic zones, Proc. Natl. Acad. Sci. 107 (2010) 17933-17938.

[44] S.E. D'Souza, M.H. Ginsberg, E.F. Plow, Arginyl-glycyl-aspartic acid (RGD): a cell adhesion motif, Trends Biochem. Sci. 16 (1991) 246-250.

[45] S. Sokic, M.C. Christenson, J.C. Larson, A.A. Appel, E.M. Brey, G. Papavasiliou, Evaluation of MMP substrate concentration and specificity for neovascularization of hydrogel scaffolds, Biomater. Sci. 2 (2014) 1343-1354.

[46] J.E. Leslie-Barbick, J.E. Saik, D.J. Gould, M.E. Dickinson, J.L. West, The promotion of microvasculature formation in poly (ethylene glycol) diacrylate hydrogels by an immobilized VEGF-mimetic peptide, Biomaterials. 32 (2011) 5782-5789.

[47] R. Lakshmanan, P. Kumaraswamy, U.M. Krishnan, S. Sethuraman, Engineering a growth factor embedded nanofiber matrix niche to promote vascularization for functional cardiac regeneration, Biomaterials. 97 (2016) 176-195.

[48] F.J. O'brien, Biomaterials \& scaffolds for tissue engineering, Mater. Today. 14 (2011) 8895.

[49] S.J. Hollister, Porous scaffold design for tissue engineering, Nat. Mater. 4 (2005) 518524.

[50] T.R. Chan, P.J. Stahl, S.M. Yu, Matrix-bound vegf mimetic peptides: design and endothelial-cell activation in collagen scaffolds, Adv. Funct. Mater. 21 (2011) 4252-4262.

[51] P.J. Stahl, T.R. Chan, Y. Shen, G. Sun, S. Gerecht, S.M. Yu, Capillary network-like organization of endothelial cells in PEGDA scaffolds encoded with angiogenic signals via triple helical hybridization, Adv. Funct. Mater. 24 (2014) 3213-3225.

[52] M.N. Nakatsu, R.C.A. Sainson, S. Pérez-del-Pulgar, J.N. Aoto, M. Aitkenhead, K.L. Taylor, P.M. Carpenter, C.C.W. Hughes, VEGF 121 and VEGF 165 regulate blood vessel diameter through vascular endothelial growth factor receptor 2 in an in vitro angiogenesis model, Lab. Investig. 83 (2003) 1873-1885.

[53] S.M. Staubli, G. Cerino, I.G. De Torre, M. Alonso, D. Oertli, F. Eckstein, K. Glatz, J.C.R. Cabello, A. Marsano, Control of angiogenesis and host response by modulating the cell adhesion properties of an Elastin-Like Recombinamer-based hydrogel, Biomaterials. 135 (2017) 30-41.

[54] S.B. Riemenschneider, D.J. Mattia, J.S. Wendel, J.A. Schaefer, L. Ye, P.A. Guzman, R.T. Tranquillo, Inosculation and perfusion of pre-vascularized tissue patches containing aligned human microvessels after myocardial infarction, Biomaterials. 97 (2016) 51-61. 